\title{
Characterization of a two-color pump-probe setup at FLASH using a velocity map imaging spectrometer
}

\author{
P. Johnsson, ${ }^{1,2, *}$ A. Rouzée, ${ }^{1}$ W. Siu,${ }^{1}$ Y. Huismans, ${ }^{1}$ F. Lépine, ${ }^{3}$ T. Marchenko, ${ }^{4,5}$ S. Düsterer,${ }^{6}$ \\ F. Tavella, ${ }^{6}$ N. Stojanovic, ${ }^{6}$ H. Redlin,${ }^{6}$ A. Azima,${ }^{6}$ and M. J. J. Vrakking ${ }^{1,7}$ \\ ${ }^{1}$ Foundation for Fundamental Research on Matter-Institute for Atomic and Molecular Physics, \\ Science Park 104, 1098 XG Amsterdam, The Netherlands \\ ${ }^{2}$ Lund University, P.O. Box 118, SE-221 oo Lund, Sweden \\ ${ }^{3}$ Université Lyon 1, CNRS, Laboratoire de Spectrométrie Ionique et Moléculaire, UMR 5579, \\ 43 boulevard du 11 novembre 1918, F-69622 Villeurbanne, France \\ ${ }^{4}$ Université Pierre et Marie Curie, Laboratoire de Chimie Physique-Matière et Rayonnement, \\ UMR 7614, 11 rue Pierre et Marie Curie, F-75005 Paris, France \\ ${ }^{5}$ CNRS, Laboratoire de Chimie Physique-Matière et Rayonnement, UMR 7614, \\ 11 rue Pierre et Marie Curie, F-75005 Paris, France \\ ${ }^{6}$ Hamburger Synchrotronstrahlungslabor (HASYLAB) at Deutsches Elektronen-Synchrotron, \\ Notkestrasse 85, D-22603 Hamburg, Germany \\ ${ }^{7}$ Max-Born-Institut, Max Born Strasse 2A, D-12489 Berlin, Germany \\ ${ }^{*}$ Corresponding author: per.johnsson@fysik.lth.se
}

Received September 10, 2010; accepted September 30, 2010; posted November 11, 2010 (Doc. ID 134858); published December 13, 2010

We report on the implementation of a high-count-rate charged particle imaging detector for two-color pump-probe experiments at the free electron laser in Hamburg (FLASH). In doing so, we have developed a procedure for finding the spatial and temporal overlap between the extreme UV free electron laser (FEL) pulses and the IR pulses, which allows for complete alignment of the setup in situations where the region of overlap between the FEL and the IR is not easily accessible by means of imaging optics. (c) 2010 Optical Society of America

OCIS codes: $140.2600,140.3298,300.6530,000.2170,220.1140$

Free electron lasers (FELs) operating in the extreme UV (XUV) and x-ray regions [1,2], featuring high photon fluxes combined with temporal and spatial coherence, are rapidly becoming important tools for investigating the structure of matter [3]. One of the great advantages of FELs over traditional synchrotron-based sources is the short pulse duration that is achievable, enabling timeresolved studies with resolutions in the few femtoseconds regime. To perform such experiments, it becomes important to implement pump-probe techniques in which the FEL pulses are synchronized with visible or IR pulses from a second laser source. Scaling pumpprobe experiments from laboratory-scale tabletop laser experiments to facility-scale FEL experiments poses new challenges, such as finding the spatial and temporal overlap as quickly as possible to utilize the limited beam time efficiently. To date, pump-probe experiments at FELs have been reported where the spatial overlap was achieved by optical imaging techniques, requiring optical access to the interaction region [4].

Here we report on an XUV/IR pump-probe experiment using a velocity map imaging spectrometer (VMIS) [5] at FLASH. The VMIS is ideally suited to perform momentum imaging of the charged particles resulting from ionization by the high flux FEL, as it allows for very high count rates $\left(10^{4}-10^{5}\right.$ events/shot) [6]. In addition, the flexibility of the VMIS, allowing it to also be used both in time-of-flight and spatial imaging modes, provides excellent opportunities for finding spatial and temporal overlap, even though the interaction region cannot be reached by optical means. The aim of this Letter is to present a step-by-step procedure for setting up two-pulse experiments at FELs, using the spectrometer itself as the alignment tool.
In the VMIS, the laser beams propagating in the $x-y$ plane are crossed with a pulsed molecular beam traveling along the detector axis $z$ and entering through a hole in the repeller electrode (see Fig. 1). By the potentials applied on the repeller $\left(V_{R}\right)$ and extractor $\left(V_{E}\right)$ electrodes, the charged particles resulting from ionization and fragmentation are accelerated into a field-free flight tube, and are ultimately detected by a microchannel plate/ phosphor screen assembly imaged by a CCD camera. Charge and mass selection for ions is made possible through the application of a $200 \mathrm{~ns}$ gate on the detector.

Depending on the relative voltages of the extractor and the repeller, $\eta=V_{E} / V_{R}$, the spectrometer can be operated either in spatial or velocity imaging mode. In

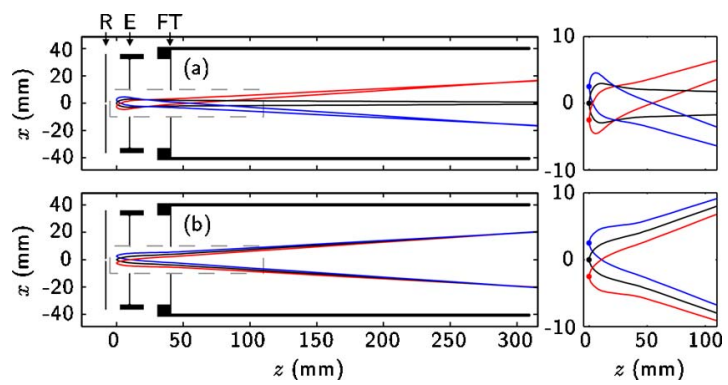

Fig. 1. (Color online) Design and calculated trajectories for the VMIS with repeller (R), extractor (E), and a grounded flight tube (FT). Positively charged particles are created at $x=0$ and $\pm 2.5 \mathrm{~mm}$ with a kinetic energy of $2 \mathrm{eV}$ in the positive and negative $x$ directions. Particle trajectories are shown for (a) spatial imaging mode and (b) velocity imaging mode, for a repeller voltage $V_{R}=1 \mathrm{kV}$. The right panels show the magnified sections marked by gray dashed rectangles. 
spatial imaging mode, a one-to-one mapping between the ionization coordinate $\left(x_{i}, y_{i}\right)$ and the impact coordinate on the detector $\left(x_{d}, y_{d}\right)$ exists, while in velocity imaging mode, the impact coordinate is more or less independent of the ionization coordinate and depends only on the initial transversal velocity of the fragments. To deduce the ionization coordinate along the detector axis $\left(z_{i}\right)$, one can use the flight time of the fragments, i.e., by measuring the total current over the phosphor screen with an oscilloscope, without gating the detector (time-offlight mode).

For the particular electrode geometry used in this experiment (shown in Fig. 1), $\eta \approx 0.98$ corresponds to the spatial imaging mode. The spatial resolution depends on the ratio between the fragment kinetic energy $\left(E_{\text {kin }}\right)$ and the voltage applied on the repeller $\left(V_{R}\right)$ while the magnification, in our case $x_{d} / x_{i}=y_{d} / y_{i} \approx-6$, depends on the spectrometer design and is independent of $V_{R}$. By trajectory calculations for a large number of ionization coordinates and kinetic energies, the achievable resolution can be estimated. As shown in Fig. 2, in spatial imaging mode, the spatial resolution in the $x-y$ plane can be optimized for a certain kinetic energy by tuning of $\eta$, but also for lower fragment energies the resolution stays reasonable. With our choice of $\eta=0.980$ sub- $100 \mu \mathrm{m}$ resolution is reached for relative fragment energies up to $2 \mathrm{eV} / \mathrm{kV}$.

Velocity imaging mode is obtained using $\eta \approx 0.77$, and repeating the trajectory calculations for this mode shows that a relative energy resolution on the order of $1 \%$ can be achieved over an energy range of several electron volts per kilovolt repeller voltage.

The experiment used the in-house IR laser at FLASH, described in detail in [7], which was focused and overlapped with the FEL beam in a noncollinear arrangement with both beams in the $x-y$ plane of the spectrometer, with an angle of $10^{\circ}$ between the two beams (see [ ] for details).

The alignment of the overlap between the FEL and the IR laser can be divided in three steps. First, a coarse temporal overlap at the experiment has to be achieved. This can, in principle, be found by using an in-vacuum photodiode that looks at both the FEL and the IR beam in the interaction region [4]. With that approach, a photodiode that is able to detect both wavelengths is needed. By using the charged particle imaging spectrometer in electron time-of-flight mode with velocity imaging settings to make the arrival time independent of $z_{i}$ and a high acceleration field so that the electron time of flight is only weakly dependent on the initial electron energy, one can instead use the arrival time of the photoelectrons on the detector as a measure of the rough timing. In our case, for $V_{R}=-10 \mathrm{kV}$ and $\eta=0.77$, the electron time

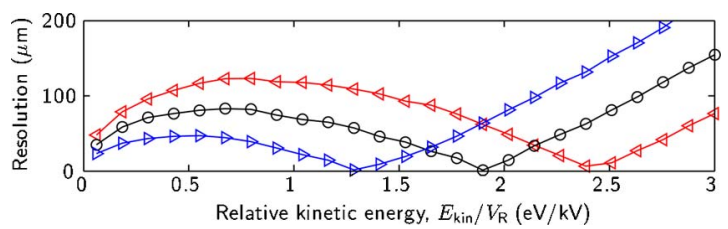

Fig. 2. (Color online) Spatial resolution estimates from trajectory calculations for $\eta=0.979$ (red $\triangleleft$ ), 0.980 (black $\bigcirc$ ), and 0.981 (blue $\triangleright$ ). of flight is $6.6 \mathrm{~ns}$ for zero energy electrons, with a spread of $\sim 500 \mathrm{ps}$ for electrons with an energy of $100 \mathrm{eV}$ ejected in opposite directions along the detector axis $z$, independent of the ionization coordinate $z_{i}$.

Once temporal overlap is ensured to within a nanosecond, the spatial overlap between the two laser beams and with the molecular beam can be found by operating the spectrometer in time-of-flight mode and spatial imaging mode. As described above, the ionization coordinate along the detector axis, $z_{i}$, can be deduced from the flight time of the ions, and overlapping the ion timeof-flight contribution from both beams ensures that $z_{i}$ is the same for the two beams. Alignment in the $x-y$ plane (detector plane) is performed using the spatial imaging mode. As an example, Figs. 3(a) and 3(b) show the detector image in spatial imaging mode. In Fig. 3(a), the molecular beam is turned off, and the detected ions come from ionization of residual gas in the spectrometer. The FEL beam is seen going from left to right with approximately constant intensity, indicative of single photon ionization. The IR beam goes from the top left to the bottom right corner of the image at a $10^{\circ}$ angle to the FEL beam. For the IR, the ionization is strongest close to the center of the image, indicative of multiphoton ionization which is localized around the focus of the beam, where the intensity is highest. Thus, the spatial imaging can also be used to locate the focus position for the IR beam.

One route toward finding the precise temporal overlap between the two lasers is to use a two-color process that requires both lasers to interact with the sample simultaneously, such as the generation of sidebands [ㄴ] $]$, requiring the delay to be scanned in small time steps until the two-color signal is found. Our approach relies on bond softening in $\mathrm{H}_{2}$ [9], which is a sequential two-color process in the sense that, to observe a signal, it is sufficient that the FEL pulse precedes the IR pulse. Briefly, the FEL pulses with a photon energy of $46 \mathrm{eV}$ efficiently ionize the $\mathrm{H}_{2}$ molecules and leave the molecular ions in the stable electronic ground state [6]. The IR pulse, arriving later, causes dissociation of the molecular ion into a neutral $\mathrm{H}$ atom and an $\mathrm{H}^{+}$ion, each with a kinetic energy of $\sim 0.5 \mathrm{eV}$. As the bond-softening process only requires a single IR photon, the IR intensity can be kept sufficiently low so as not to ionize the molecules without the FEL present. Figure 4 shows experimental images recorded in velocity imaging mode, with a 200 ns gate on the detector selecting only $\mathrm{H}^{+}$ions. Figure 4(a) shows the $\mathrm{H}^{+}$distribution when the IR pulse, which by itself does not produce any $\mathrm{H}^{+}$ions, comes before the FEL pulse. It contains contributions from the $1 s \sigma_{g}^{+}$state (central peak)

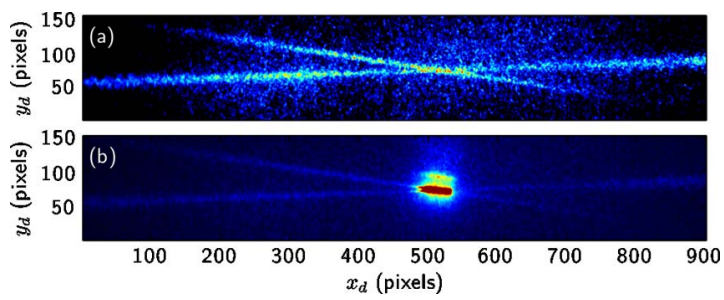

Fig. 3. (Color online) Spatial images of the interaction region with both the FEL and IR present (a) without and (b) with a molecular beam of $\mathrm{H}_{2}$. 


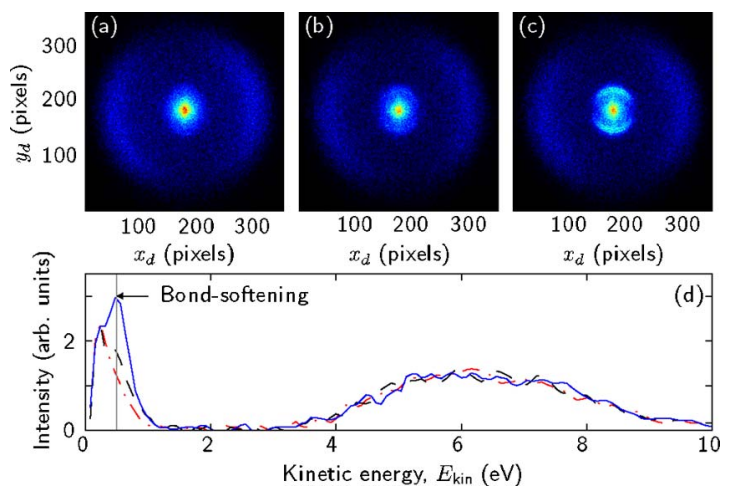

Fig. 4. (Color online) (a)-(c) Velocity images with the detector gated on $\mathrm{H}^{+}$ions from ionization of $\mathrm{H}_{2}$ by both the FEL and the IR pulses for different delays. Panel (d) shows the fragment kinetic energy distribution for $\mathrm{H}^{+}$at the same delays. (a) IR before FEL (red dashed-dotted curve), (b) at the temporal overlap (black dashed curve), and (c) FEL before IR (blue solid curve).

and the repulsive $2 p \pi_{u}$ state (outer ring). As the temporal delay is scanned across the overlap [Figs. $\underline{4(b)}$ and $\underline{4(c)}$ ], $\mathrm{H}^{+}$ions ejected along the laser polarization direction appear at low energies [see also Fig. 4(d)], as a result of dissociation through bond softening. Starting from a situation where the IR pulse comes a few nanoseconds after the FEL pulse (verified by the electron time-of-flight measurements described above) and where the bondsoftening signal is visible, it is easy to change the delay back and forth around the temporal overlap (where the bond-softening signature disappears) with decreasing step sizes and, thus, narrow down on the position of the precise temporal overlap.

In conclusion, we have given a step-by-step procedure for setting up XUV/IR pump-probe experiments at FELs using a VMIS. The described procedure has been the key tool in two recent experiments by the authors on laserinduced molecular alignment [8] and molecular dissociation [10] at FLASH.

This work is part of the research program of the "Stichting voor Fundamenteel Onderzoek der Materie (FOM)," which is financially supported by the "Nederlandse organisatie voor Wetenschappelijk Onderzoek (NWO)." P. Johnsson acknowledges the support of the Swedish Research Council and the Swedish Foundation for Strategic Research. Portions of this research were carried out at the light source facilities FLASH at HASYLAB/DESY. We acknowledge travel support from the European Community under Contract RII3CT-2004-506008 (IA-SFS) and are grateful to Rob Kemper, Ad de Snaijer, and Hinco Schoenmaker for their technical support.

\section{References}

1. W. Ackermann, G. Asova, V. Ayvazyan, A. Azima, N. Baboi, J. Bähr, V. Balandin, B. Beutner, A. Brandt, A. Bolzmann, R. Brinkmann, O. I. Brovko, M. Castellano, P. Castro, L. Catani, E. Chiadroni, S. Choroba, A. Cianchi, J. T. Costello, D. Cubaynes, J. Dardis, W. Decking, H. DelsimHashemi, A. Delserieys, G. Di Pirro, M. Dohlus, S. Düsterer, A. Eckhardt, H. T. Edwards, B. Faatz, J. Feldhaus, K. Flöttmann, J. Frisch, L. Fröhlich, T. Garvey, U. Gensch,
Ch. Gerth, M. Görler, N. Golubeva, H.-J. Grabosch, M. Grecki, O. Grimm, K. Hacker, U. Hahn, J. H. Han, K. Honkavaara, T. Hott, M. Hüning, Y. Ivanisenko, E. Jaeschke, W. Jalmuzna, T. Jezynski, R. Kammering, V. Katalev, K. Kavanagh, E. T. Kennedy, S. Khodyachykh, K. Klose, V. Kocharyan, M. Körfer, M. Kollewe, W. Koprek, S. Korepanov, D. Kostin, M. Krassilnikov, G. Kube, M. Kuhlmann, C. L. S. Lewis, L. Lilje, T. Limberg, D. Lipka, F. Löhl, H. Luna, M. Luong, M. Martins, M. Meyer, P. Michelato, V. Miltchev, W. D. Möller, L. Monaco, W. F. O. Müller, O. Napieralski, O. Napoly, P. Nicolosi, D. Nölle, T. Nuñez, A. Oppelt, C. Pagani, R. Paparella, N. Pchalek, J. Pedregosa-Gutierrez, B. Petersen, B. Petrosyan, G. Petrosyan, L. Petrosyan, J. Pflüger, E. Plönjes, L. Poletto, K. Pozniak, E. Prat, D. Proch, P. Pucyk, P. Radcliffe, H. Redlin, K. Rehlich, M. Richter, M. Roehrs, J. Roensch, R. Romaniuk, M. Ross, J. Rossbach, V. Rybnikov, M. Sachwitz, E. L. Saldin, W. Sandner, H. Schlarb, B. Schmidt, M. Schmitz, P. Schmüser, J. R. Schneider, E. A. Schneidmiller, S. Schnepp, S. Schreiber, M. Seidel, D. Sertore, A. V. Shabunov, C. Simon, S. Simrock, E. Sombrowski, A. A. Sorokin, P. Spanknebel, R. Spesyvtsev, L. Staykov, B. Steffen, F. Stephan, F. Stulle, H. Thom, K. Tiedtke, M. Tischer, S. Toleikis, R. Treusch, D. Trines, I. Tsakov, E. Vogel, T. Weiland, H. Weise, M. Wellhöfer, M. Wendt, I. Will, A. Winter, K. Wittenburg, W. Wurth, P. Yeates, M. V. Yurkov, I. Zagorodnov, and K. Zapfe, Nat. Photon. 1, 336 (2007).

2. P. Emma, R. Akre, J. Arthur, R. Bionta, C. Bostedt, J. Bozek, A. Brachmann, P. Bucksbaum, R. Coffee, F.-J. Decker, Y. Ding, D. Dowell, S. Edstrom, A. Fisher, J. Frisch, S. Gilevich, J. Hastings, G. Hays, Ph. Hering, Z. Huang, R. Iverson, H. Loos, M. Messerschmidt, A. Miahnahri, S. Moeller, H.-D. Nuhn, G. Pile, D. Ratner, J. Rzepiela, D. Schultz, T. Smith, P. Stefan, H. Tompkins, J. Turner, J. Welch, W. White, J. Wu, G. Yocky, and J. Galayda, Nat. Photon. 4, 641 (2010).

3. R. Neutze, R. Wouts, D. van der Spoel, E. Weckert, and J. Hajdu, Nature 406, 752 (2000).

4. P. Radcliffe, S. Düsterer, A. Azima, W. B. Li, E. Plönjes, H. Redlin, J. Feldhaus, P. Nicolosi, L. Poletto, J. Dardis, J. P. Gutierrez, P. Hough, K. G. Kavanagh, E. T. Kennedy, H. Lunac, P. Yeates, A. Costello, J. T. Delyseries, C. L. S. Lewis, D. Glijer, D. Cubaynes, and M. Meyer, Nucl. Instrum. Methods Phys. Res. Sect. A 583, 516 (2007).

5. A. T. J. B. Eppink and D. H. Parker, Rev. Sci. Instrum. 68, 3477 (1997).

6. P. Johnsson, W. Siu, A. Gijsbertsen, J. Verhoeven, A. S. Meijer, W. van der Zande, and M. J. J. Vrakking, J. Mod. Opt. 55, 2693 (2008).

7. H. Redlin, A. Al-Shemmary, A. Azima, N. Stojanovic, F. Tavella, I. Will, and S. Düsterer, "The FLASH pumpprobe laser system: setup, characterization and optical beamlines," Nucl. Instrum. Methods Phys. Res. Sect. A (to be published).

8. P. Johnsson, A. Rouzée, W. Siu, Y. Huismans, F. Lépine, T. Marchenko, S. Düsterer, F. Tavella, N. Stojanovic, A. Azima, R. Treusch, M. F. Kling, and M. J. J. Vrakking, J. Phys. B 42, 134017 (2009).

9. P. H. Bucksbaum, A. Zavriyev, H. G. Muller, and D. W. Schumacher, Phys. Rev. Lett. 64, 1883 (1990).

10. A. Rouzée, P. Johnsson, Y. Huismans, W. Siu, H. Fukuzawa, S. Düsterer, F. Tavella, N. Stojanovic, R. Treusch, K. Ueda, and M. J. J. Vrakking are preparing a manuscript to be called "Time-resolved photodissociation of $\mathrm{Br}_{2}$ molecules probed by XUV at FLASH." 